\title{
A CONTRIBUIÇÃO DO \\ PENSAMENTO DE SISTEMAS \\ PARA A EDUCAÇÃO AMBIENTAL: \\ TEORIA, METODOLOGIAS, \\ MÉTODOS E FERRAMENTAS
}

Cláudio Pereira de Sampaio ${ }^{1}$

Aguinaldo dos Santos ${ }^{2}$

Resumo: O potencial de compreensão dos problemas de sustentabilidade de forma integrada, sistêmica e interdisciplinar propiciado pelo pensamento de sistemas (systems thinking) se apresenta como uma promissora abordagem para a Educação Ambiental. Neste artigo são apresentadas e discutidas as principais correntes teórico-metodológicas de pensamento de sistemas funcionalistas-estruturalistas, interpretativistas e de sistemas críticos, bem como abordagens integradoras. Como resultado, propõe-se um quadro-síntese destas abordagens, com indicação da sua possível contribuição em estudos e projetos em Educação Ambiental.

Palavras-chave: Sustentabilidade; Problemas Complexos; Pensamento de Sistemas, Metodologias.

Abstract: The potential for understanding the sustainability problems of integrated, systemic and interdisciplinary way provided by systems thinking (systems thinking) presents itself as a promising approach to Environmental Education. This article presents and discusses the main theoretical and methodological currents of systems thinking - functionalist-structuralist, interpretive and critical systems, as well as integrative approaches. As a result, a summary table of these approaches is proposed, indicating their possible contribution to studies and projects in Environmental Education.

Keywords: Sustainability; Complex Problems; Systems Thinking; Methodologies.

\footnotetext{
1Universidade Estadual de Londrina (UEL). E-mail: claudiopereira@uel.br,

Link para o Lattes: http://lattes.cnpq.br/0193236846556503

2 Universidade Federal do Paraná (UFPR). E-mail: asantos@ufpr.br.

Link para o Lattes: http://lattes.cnpq.br/7834194247765889
} 


\section{Introdução: Sustentabilidade e problemas complexos}

Os problemas de sustentabilidade apresentam uma dificuldade de enfrentamento dada a sua característica de multidimensionalidade (social, econômica, ambiental, cultural, política, legal) e complexidade (múltiplas e variadas interrelações entre os elementos, e em diferentes níveis), sendo, portanto, problemas essencialmente sistêmicos, ou seja, relativos a sistemas (MURPHY, 2012). Tal sistemicidade demanda uma abordagem interdisciplinar, integrada e participativa. Outra característica é a dinamicidade (mudança ao longo do tempo) dos problemas de sustentabilidade, o que frequentemente dificulta sua definição exata e inequívoca. São problemas frequentemente mal definidos e com múltiplos ciclos de causa e efeito, além de incluir uma diversidade de stakeholders cujos interesses e pontos de vista precisam ser considerados; são os chamados wicked problems (RITTEL, WEBBER, 1968).

Essas características de sistemicidade e dinamicidade exigem formas de articulação, tanto na pesquisa quanto na intervenção, que possibilitem aos pesquisadores irem além dos seus campos disciplinares, aproximando-se mais da realidade e integrando aqueles que são afetados pelo problema estudado. Neste sentido, serão apresentadas e discutidas neste artigo as contribuições de uma área integradora do conhecimento que muito pode contribuir para o enfrentamento dos problemas reais de sustentabilidade, e em especial para a Educação Ambiental: o pensamento de sistemas.

\section{Metodologia}

Este estudo teve como recurso metodológico principal a revisão bibliográfica assistemática (RBA), principalmente com base em artigos de revistas internacionais relacionadas à área de teoria de sistemas, bem como livros de referência nestas áreas, apoiada pelo uso da plataforma digital Mendeley. O levantamento feito por meio da RBA permitiu ao autor compreender as principais abordagens de pensamento de sistemas e suas possíveis aplicações em estudos e projetos de Educação Ambiental. A seguir é apresentada uma descrição sintética dos principais aspectos teóricometodológicos identificados na literatura, seguida da proposição de um quadrosíntese que permite visualizar estes aspectos, como decorrência de estudo anterior feito por Sampaio (2019).

\section{Os problemas de sustentabilidade: ontologia e epistemologia}

A complexidade dos problemas de sustentabilidade, aqui consideradas como problemas de sistemas (ou sistêmicos), pode ser abordada com base em uma polaridade ontológica entre ordem e não-ordem ("un-order", no sentido de ordem emergente, diferente de "disorder", desordem), o que nos leva a três categorias de análise (SNOWDEN, 2005): Ordem, complexidade e caos. Snowden (idem) propõe agrupar estes dois últimos em uma única categoria, 
para fins de análise. A partir destas três categorias, podemos identificar pelo menos quatro tipos de problemas relativos a sistemas (ALMAN, 2013):

- Problemas simples: com alto nível de ordem, relações de causa e efeito são diretas e observáveis (Ex. sistemas mecânicos, motores);

- Problemas complicados: com alto ou médio nível de ordem, relações previsíveis, mas indiretas de causa-efeito (Ex. sistemas industriais);

- Problemas complexos: com baixo nível de ordem, relações imprevisíveis e nas quais as relações causais podem ser vistas apenas de forma retrospectiva (Ex. sistemas sociotécnicos, como coleta seletiva municipal);

- Problemas caóticos: com nível de ordem emergente, não se pode identificar nenhum sistema operando, o que exige gestão de crises e decisões adhoc (Ex. sistemas de previsão climática).

Para o enfrentamento de cada um destes tipos de problemas pode-se adotar posturas e abordagens distintas, o que nos leva à dimensão epistemológica, que se refere à forma pela qual podemos compreender e agir em relação àquilo que estamos buscando conhecer. Assim, e partindo-se do raciocínio sistêmico, pode-se adotar para os problemas de sustentabilidade ao menos três perspectivas distintas: unitarista (entendimento homogêneo e unificado sobre o problema), pluralista (entendimento heterogêneo e variado) e coercitiva (entendimento heterogêneo, variado e emancipatório). Estas três perspectivas estão diretamente relacionadas às principais correntes e metodologias de pensamento de sistemas, as quais serão discutidas a seguir.

\section{Pensamento de sistemas}

Neste estudo buscou-se identificar apenas abordagens sistêmicas que oferecessem possibilidades pragmáticas em termos de metodologias, métodos e ferramentas para estudos e projetos em sustentabilidade. Por este motivo, não foi incluída a abordagem de sistemas complexos, a qual, embora apresente conceitos e princípios úteis como controle distribuído, emergência, conectividade, coevolução e estado de paradoxo (MILLER; PAGE, 2007), não oferece recursos práticos/metodológicos para a operacionalização destes conceitos. Apesar disso, cabe ressaltar que a complexidade é um conceito fundamental para a sustentabilidade e para algumas abordagens filosóficas citadas neste artigo, em especial o Estruturalismo (que considera o fenômeno da emergência) e o Realismo Crítico, aqui citado como exemplo de perspectiva filosófica integrativa.

Cabe aqui também definir o que seja um sistema. Em termos ontológicos, Ison (2010) define que "(um sistema) é um todo integrado do qual as propriedades essenciais emergem das relações entre as partes que 0 compõem". Já sob uma perspectiva epistemológica, "um sistema é um todo, uma entidade que um observador pode cognitivamente dissociar de um 
contexto", e "exibe uma certa forma de organização que determina seu caráter individual" (VANDENBROECK, 2015). O pensamento de sistemas (Systems Thinking ou Systems Science) caracteriza-se como um campo interdisciplinar de conhecimento que envolve pesquisadores tanto oriundos das ciências naturais (as chamadas "hard sciences") quanto das ciências sociais ("soft sciences"), e também daquelas áreas que situam-se na interface do mundo natural e social, às quais Simon (1969) denominou "Ciências do Artificial" (arquitetura, engenharia, design, planejamento urbano). Esta divisão das ciências influenciou diretamente o modo como se desenvolveu o pensamento de sistemas ao longo do século XX até agora, levando a uma polarização entre o chamado "hard systems thinking" e o "soft systems thinking".

\section{Hard Systems: Sistemas funcionalistas e estruturalistas}

A primeira corrente do pensamento de sistemas denominado "hard systems", de caráter funcionalista, foi influenciada tanto pelo positivismo científico, então dominante no início do século $X X$, quanto pela revolução industrial ocorrida no final do século XIX e começo do século XX; com sua necessidade de otimização dos processos e eliminação de desperdícios, a Administração Científica de Taylor (ou Taylorismo) foi a base teóricometodológica mais proeminente deste período (PORTER; CÓRDOBA, 2009). Embora já no final dos anos 20 fosse considerada ultrapassada, vários dos seus princípios permanecem ainda relevantes, e influenciaram teorias posteriores, como a Engenharia de Sistemas (Systems Engineering, ou SE) e a Pesquisa Operacional (Operations Research, ou OR).

A segunda corrente dos "hard systems" emergiu a partir dos anos 40, com estudos que buscavam combinar conhecimentos das ciências naturais com os estudos sobre os indivíduos, organizações e tecnologias. Tal combinação resultou, conforme citado por Lazslo e Krippner (1998), Porter e Córdoba (2009) e Mingers e White (2009), em um conjunto bastante variado de teorias, entre elas a Teoria Geral dos Sistemas (BERTALANFY, 1950), a Cibernética (ASHBY, 1956; WIENER, 1954), a Dinâmica de Sistemas (FORRESTER, 1968), o Modelo de Sistema Viável (BEER, 1967), e nos anos $70 \mathrm{com}$ a Autopoiesis (MATURANA, VARELA, 1979), além das teorias sobre estrutura social de Parsons (1951) e Bateson (1972), entre outros. No caso específico da Dinâmica de Sistemas (System Dynamics), a criação de modelos matemáticos computacionais para simulação do comportamento dinâmico de sistemas é uma das contribuições mais importantes desta corrente, e que resultou em estudos como o Limites do Crescimento (MEADOWS et al., 1972), publicado em 1971 pelo Clube de Roma.

Ambas as abordagens - funcionalista e estruturalista - compartilham a

percepção dos sistemas como sendo estruturas objetivas, reais, as quais podem ser conhecidas por meio dos métodos e processos corretos e otimizadas para um funcionamento ideal. Adota-se aqui, portanto, uma perspectiva unitarista, no sentido de que há uma percepção ideal do sistema,

revista brasileira educação ambiental 
normalmente obtida a partir do consenso de especialistas. Porém, enquanto os sistemas funcionalistas são vistos como resultado da soma das funções entre suas partes e essencialmente fechados, os estruturalistas consideram a abertura e troca com o ambiente como característica importante para 0 comportamento dinâmico, no qual o sistema resulta das interações, e não apenas da soma dos seus elementos.

As abordagens funcionalistas e estruturalistas são bastante úteis em estudos e projetos de sustentabilidade nos quais se busca compreender os aspectos objetivos ligados aos fenômenos naturais (Ex. impactos ambientais) e os aspectos técnicos relativos às formas de exploração, produção, distribuição e descarte de produtos e serviços, ou seja, às diversas tecnologias, incluindo a gestão destas atividades. Tal compreensão pode levar a uma intervenção que busque o melhor funcionamento do sistema, a melhoria dos fluxos e interações, a otimização no uso de recursos, o uso de recursos menos impactantes, a melhor adequação entre vida útil e durabilidade esperada, ou mesmo a melhores tecnologias de descarte e revalorização dos materiais. Para isso, há uma grande variedade de metodologias, métodos e ferramentas, tanto de caráter quantitativo quanto qualitativo. Há inclusive heurísticas (diretrizes, regras de ação, recomendações) de sistemas que podem ser úteis tanto para uma melhor caracterização dos sistemas, como os arquétipos de sistemas (KIM, 1992), quanto para definição de ações preferenciais por meio da identificação de pontos de alavancagem de sistema (MEADOWS, 1999). Tais heurísticas podem ser utilizadas como norteadores de ações em projetos e atividades de Educação Ambiental que buscam ampliar a compreensão sobre como ampliar a eficiência do sistema sob estudo e/ou intervenção.

Os arquétipos de sistemas representam modelos de sistema com padrões de comportamento mais comuns, como "Metas à Deriva", "Escalonamento", "Consertos que Falham", "Limites para o Sucesso", "Tragédia dos Bens Comuns", entre outros (KIM, 1992). Os pontos de alavancagem, por sua vez, são os lugares do sistema nos quais pequenas mudanças podem gerar mudanças significativas. Meadows (1999) propõe doze pontos de alavancagem em ordem crescente de potencial de mudança: 1) Constantes, parâmetros e números, 2) tamanho das reservas, 3) estruturas de estoque e fluxo, 4) atrasos relativos a taxas de mudança, 5) ciclos de feedback, 6) estruturas de fluxo de informação, 7) regras do sistema, 8) auto-organização do sistema, 9) objetivo do sistema, 10) paradigma utilizado para criar o sistema, e 12) força para mudar o paradigma ao lidar com novos desafios. Estes paradigmas alternativos podem incluir outras abordagens de sistemas como os soft systems, a seguir.

\section{Soft Systems: Sistemas interpretativistas}

A constatação das limitações das abordagens funcionalistas e estruturalistas em lidar com as questões mais subjetivas relativas ao componente humano dos sistemas levou, a partir da década de 1960, ao 
surgimento de uma corrente de pensamento de sistemas denominada soft systems. Ao incluir teorias sociais advindas de fontes como a Psicologia, Psicanálise e a Teoria Crítica da Escola de Frankfurt (Horkheimer, Adorno, Habermas e outros), aliada a uma observação direta da realidade social, esta corrente apresenta forte caráter interpretativista, ou seja, compreende os sistemas como representações mentais dos indivíduos que compõem uma determinada realidade. O objetivo aqui é, portanto, a realidade plural, socialmente construída, mais do que a sua representação objetiva e unitarista. A participação, a colaboração, o compartilhamento e o aprendizado assumem um papel central nas abordagens interpretativistas de sistemas, aqui vistos como essencialmente abertos em relação ao ambiente, embora necessitem ser delimitados artificialmente para fins de estudos. Destaca-se nesta corrente a metodologia Soft Systems Methodology (SSM).

\section{Soft Systems Methodology (SSM)}

Desenvolvida nos anos 1970 por Checkland (1990) com o objetivo de facilitar processos participativos de análise, criação, gestão e tomada de decisão sobre problemas complexos em organizações e contextos sociais, a SSM tem como. Princípios: 1) propósito das atividades; 2) visão de mundo dos atores; 2) SSM como um processo de aprendizagem; e 4) uso de modelos conceituais como modelos informacionais. A SSM inicialmente teve influência da abordagem de engenharia de sistemas (systems engineering), o que levou seu autor a elaborar um modelo estruturado de sete fases (ibidem):

1. Compreender o problema por meio de Rich Pictures e outras ferramentas;

2. Estruturar a situação, identificando sistemas humano-atividade relevantes e intencionais por meio de diagramas de transformação InputOutput;

3. Clarificar o sistema, por meio da definição de definições-raiz (root definitions) e de um framework denominado CATWOE (Customer-ActorTransformation-Worldview-Owner-Environment);

4. Elaborar de forma participativa modelos conceituais do sistema, com as atividades requeridas pelos atores;

5. Comparar os modelos conceituais com a situação real;

6. Analisar a viabilidade (cultural, técnica, dependências, decisões ganhaganha), prioridades e riscos da mudança;

7. Implementar ou facilitar as mudanças, com um plano de intervenção.

Este modelo foi posteriormente sintetizado em quatro atividades principais:

1. Compreender o problema, considerando aspectos culturais e políticos;

2. Elaborar modelos de sistema humano-atividade relevantes e intencionais;

3. Debater com os atores envolvidos, apoiado por modelos conceituais;

4. Agir para promover mudanças e melhorar a situação. 
A abordagem de soft systems, com destaque para a SSM, oferece para os projetos e atividades de Educação Ambiental uma perspectiva mais inclusiva de construção da realidade em estudo e/ou intervenção, especialmente em situações em que haja um relativo equilíbrio de poder (Ex. sociedades ou organizações democraticamente maduras). No entanto, também apresenta fragilidades em termos de como lidar adequadamente com situações onde há coerção e/ou falta de condições de manifestação de opinião adequada para uma parcela dos atores, que acabam sendo segregados do processo de decisão. Para lidar com este tipo de situação foi desenvolvido o Pensamento Crítico de sistemas (Critical Systems Thinking).

\section{Critical Systems: Os Sistemas Críticos}

O Pensamento Crítico de Sistemas (Critical Systems Thinking, ou CST) foi inicialmente desenvolvido por Churchman (1971, apud ULRICH, 2010), e posteriormente por Ulrich (1983) e Flood e Jackson (1991), entre outros. Os princípios norteadores do CST são 1) consciência crítica; 2) consciência social; 3) complementaridade ao nível das metodologias, métodos e ferramentas; 4) complementaridade teórica entre as abordagens de sistemas; e 5) emancipação humana (LAZLO; KRIPPNER, 1998). Em termos práticos, esta abordagem pode ser operacionalizada por meio das Heurísticas de Sistemas Críticos (Critical Systems Heuristics, ou CSH) desenvolvidas por Ulrich (1983), e que incluem doze questões críticas, conforme o Quadro 1.

\section{Quadro 1: Questões Norteadoras da CSH}

MOTIVAÇÃO

1. Quem é o cliente/beneficiário do sistema a ser aprimorado?

2. Qual deve ser o propósito do Sistema?

3. Qual deve ser a medida de sucesso ou aprimoramento do sistema? CONTROLE

Quem é o tomador de decisão, ou seja, quem tem poder alterar as medidas de sucesso?

Que componentes do sistema são controlados pelo tomador de decisão?

Que recursos e condições são parte do ambiente, e não devem ser controlados apenas pelo decisor?

\section{EXPERTISE}

Quem deve ser envolvido como criador do sistema?

Que tipo de expertise deve ser inserido na criação do sistema, quem a detém e deveria participar, e qual o seu papel?

Quem deve garantir que as melhorias sejam implementadas e consideradas bem-sucedidas de acordo com as medidas definidas inicialmente?

\section{LEGITIMAÇÃO}

Quem deve representar os interesses dos cidadãos que serão afetados pelo novo sistema? Quem deve participar?

Em que extensão e de que modo os afetados têm a possibilidade de emancipação das premissas e promessas dos envolvidos?

11. Sobre quais perspectivas da realidade, e de quem, as mudanças se assentam?

Fonte: Traduzido de Ulrich (1983). 
A abordagem de critical systems oferece para estudos e projetos em sustentabilidade, e aqui especialmente para projetos e atividades em Educação Ambiental, a possibilidade de apreciação dos aspectos humanos, tanto individuais quanto sociais, de caráter mais subjetivo e interpretativo, por meio de uma abordagem participativa e colaborativa, mas além disso buscando a emancipação de grupos sociais em situações de coerção. Tal situação não é incomum no Brasil, portanto esta é uma abordagem particularmente útil para a realidade brasileira, e que pode ainda ser combinada com a Educação Ambiental Crítica-Emancipatória (EAE) para fortalecer a formação de valores e conceitos desde a infância. Neste sentido, a utilização das CSH em projetos e atividades de EAE pode ajudar a ampliar e aprofundar a compreensão do contexto de estudo e/ou intervenção.

\section{Abordagens integradoras de sistemas}

A integração das diferentes abordagens de sistemas têm sido objeto de estudo desde a década de 70, e a seguir destacamos duas propostas, ambas surgidas a partir da teoria de sistemas críticos: Total Systems Intervention (TSI) e Multimetodologia.

\section{Total Systems Intervention (TSI)}

Esta proposta foi elaborada por Flood (1991), a partir de quatro princípios: 1) inteireza da realidade, estratificada em níveis (influência da filosofia do realismo Crítico de Roy Bhaskar); 2) participação significativa dos envolvidos e afetados pelo sistema; 3 ) reflexão sobre os interesses organizacionais e dominância de determinados métodos; 4) liberdade humana, possibilitada pela participação reflexiva. A TSI foi estruturada metodologicamente em três fases: 1) Criatividade, onde se explora a realidade onde haverá intervenção; 2) Escolha, onde se escolhem os métodos mais adequados aos objetivos da intervenção; e 3) Implementação, onde os métodos são aplicados a fim de se criar e implementar as mudanças propostas de forma participativa.

\section{Multimetodologia}

A preocupação em como combinar métodos e ferramentas oriundos das diferentes abordagens de sistemas levou Mingers e Brocklesby (1997) a desenvolver um framework que possibilita esta atividade, considerando-se três dimensões: material, pessoal e social, ao longo das fases ou atividades de um determinado estudo ou projeto. Estas fases, baseadas na SSM, são quatro: Apreciação, Análise, Desenvolvimento e Ação.

As estruturas integradoras destas duas propostas metodológicas podem ser utilizadas para enriquecer projetos e atividades de Educação Ambiental mais complexas em que haja a necessidade de se lidar ao mesmo tempo com aspectos relativos ao mundo natural e ao mundo humano, incluindo-se nesta interação a tecnologia, a economia e outros temas relevantes. 
Quadro 2: síntese das abordagens de pensamento de sistemas e sua possível contribuição para estudos e projetos em Educação Ambiental. Fonte: Elaborado pelo autor.

Quadro 2: síntese das abordagens de pensamento de sistemas e sua possível contribuição para estudos e projetos em Educação Ambiental. Fonte: Elaborado pelo autor. 


\section{Conclusões}

Pretende-se que o estudo das abordagens de pensamento de sistemas aqui apresentado seja utilizado como referencial teórico e metodológico para a elaboração de novos modelos de estudo e/ou intervenção em Educação Ambiental, e adaptados à realidade brasileira em nível local e regional. Os conceitos, princípios, metodologias, métodos e ferramentas disponíveis para cada uma das abordagens pode ser incorporado à Educação Ambiental conforme o objetivo desta e a natureza do problema abordado, o que em grande parte determina o caminho epistemológico a ser adotado, como visto neste artigo. Neste sentido, e como contribuição final, o Quadro 2 (próxima págna) apresenta uma síntese das abordagens de pensamento de sistemas apresentadas neste artigo, e sua possível contribuição em estudos e projetos voltados à Educação Ambiental.

A primeira consideração a ser feita refere-se às bases filosóficas, as quais permitem situar as diversas perspectivas de pensamento de sistemas em termos dos paradigmas conceituais nos quais se apoiam, sejam eles de caráter estritamente funcionalista-positivista, estruturalista, interpretativista ou mesmo crítico, este último com forte influência do materialismo dialético. Tais bases incluem, inclusive, uma possibilidade integrativa, sistêmica, complexa e nãoexcludente de paradigmas, dada pelo Realismo Crítico de Roy Bhaskar.

Cada um destes paradigmas considera o sistema em termos ontológicos distintos, seja no objetivismo reducionista que considera o sistema em termos da soma das funções dos seus elementos integrantes, na visão sinérgica do sistema como resultado das interações entre esses elementos, até a consideração deste como resultado das percepções individuais e coletivas da realidade, muitas vezes em situações de assimetria de poder. Aqui também o Realismo Crítico apresenta uma perspectiva ontológica que alia a objetividade dos sistemas e a subjetividade dos indivíduos dele participantes.

Estas perspectivas ontológicas direcionam também a epistemologia adotada, a qual pode se dar tanto a partir de uma visão unitarista e homogênea, no caso dos sistemas funcionalistas e estruturalistas, como também pela pluralidade de ideias, percepções, expectativas e intenções dos diversos atores que compõem esse sistema. Esta pluralidade epistemológica é característica fundamental também de metodologias integradoras de sistemas como a TSI e a Multimetodologia. 


\begin{tabular}{|c|c|c|c|c|c|}
\hline 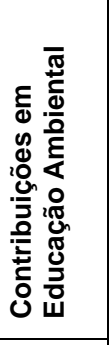 & 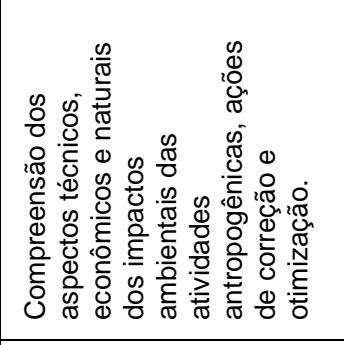 & 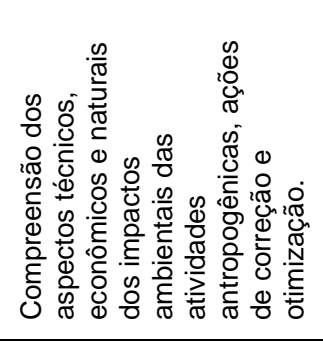 & 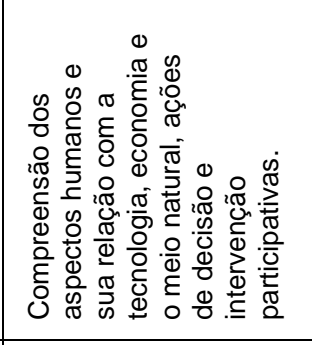 & 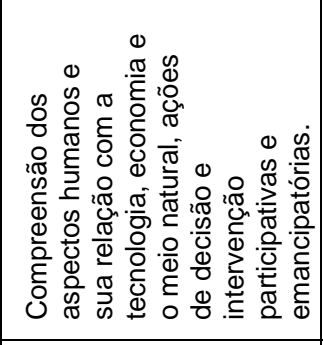 & 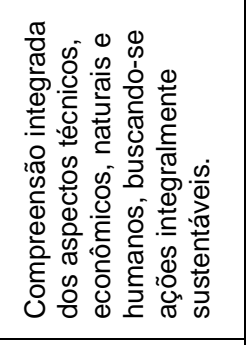 \\
\hline 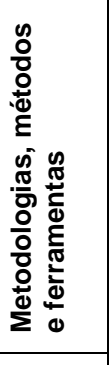 & 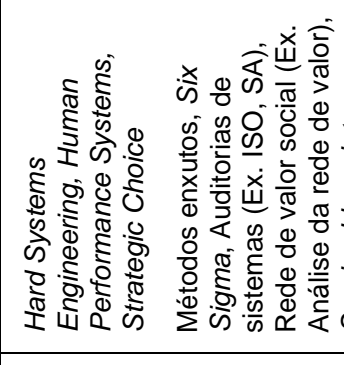 & 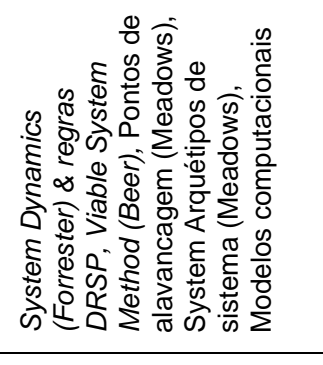 & 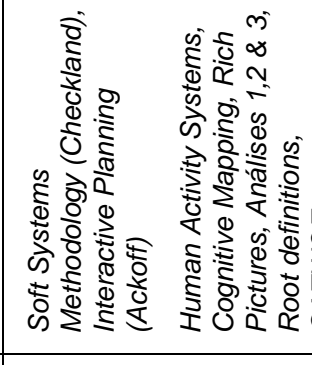 & 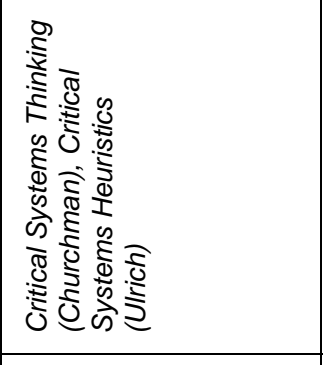 & 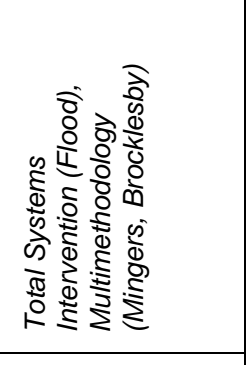 \\
\hline 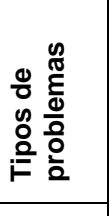 & 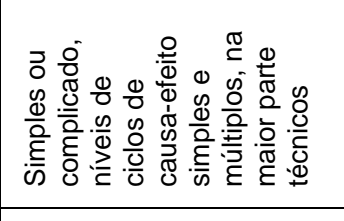 & 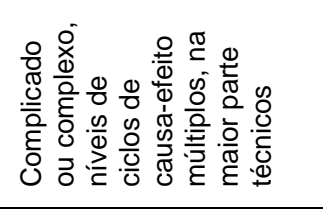 & 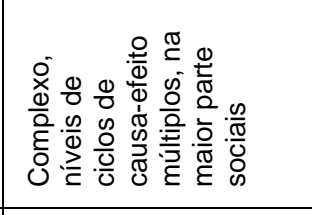 & 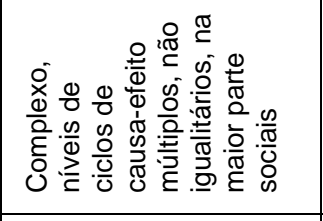 & $\begin{array}{l}\text { : } \\
\text { 量 }\end{array}$ \\
\hline 高 & 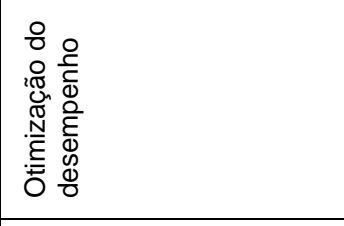 & 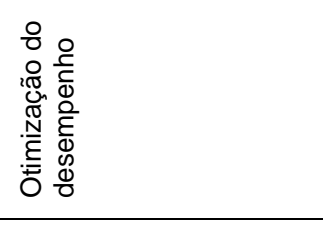 & 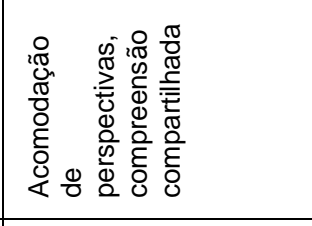 & 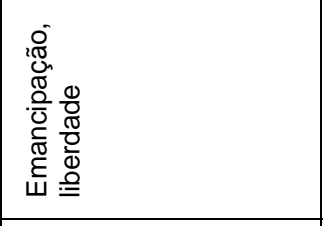 & $\begin{array}{l}\text { 总 } \\
\text { 妾 }\end{array}$ \\
\hline 吕 & 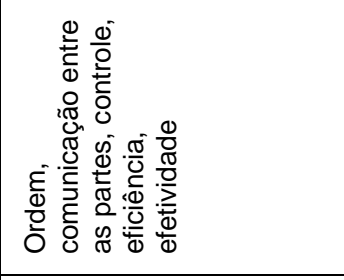 & 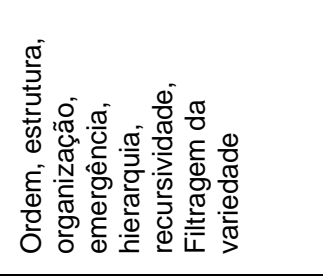 & 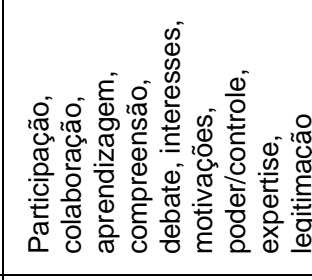 & 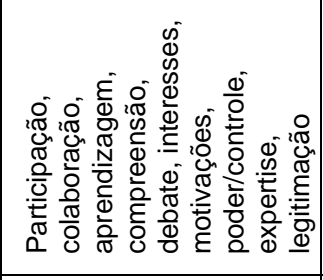 & $\begin{array}{l}\text { 总 } \\
\text { 妾 }\end{array}$ \\
\hline 㯊 & 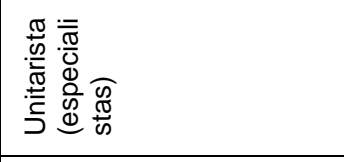 & 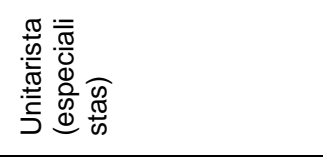 & 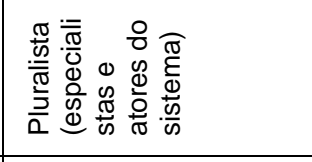 & 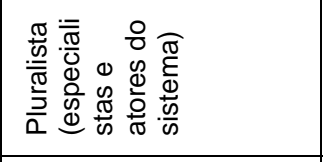 & 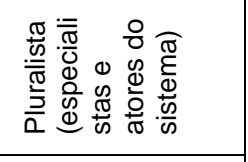 \\
\hline 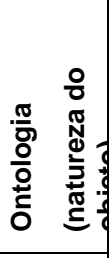 & 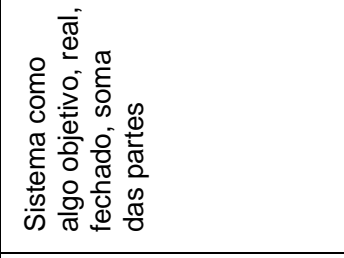 & 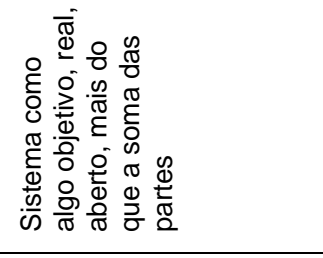 & 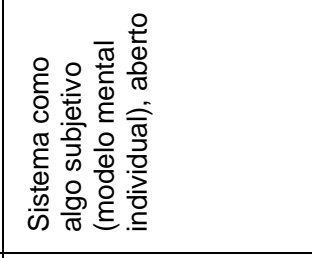 & 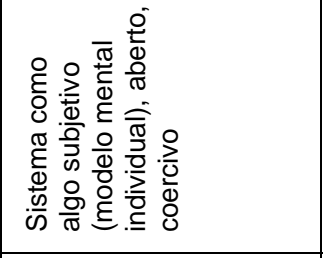 & 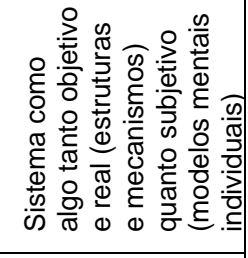 \\
\hline 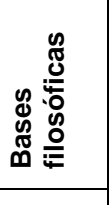 & 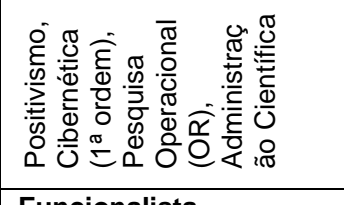 & 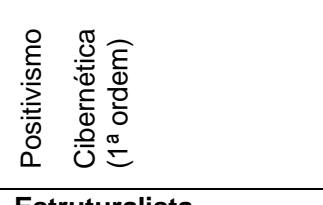 & 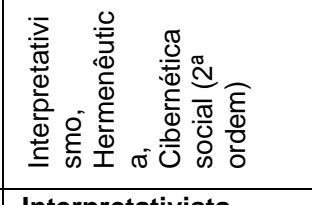 & 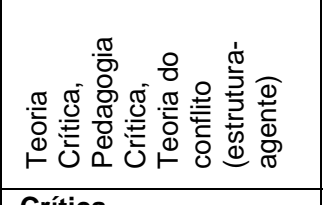 & 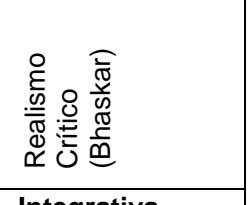 \\
\hline & Funcionalista & Estruturalista & Interpretativista & Crítica & Integrativa \\
\hline
\end{tabular}


O princípio norteador dos sistemas funcionalistas e estruturalistas é o da busca de ordem e eficiência, embora estes últimos considerem a possibilidade de comportamentos emergentes que podem alterar estruturalmente o sistema; já no caso dos sistemas interpretativistas e críticos há uma ênfase mais participativa, colaborativa e centrada na aprendizagem a fim de promover eventuais consensos, ou mesmo evidenciar a sua falta.Tanto a necessidade de ordem e eficiência quanto de participação e colaboração caracterizam as metodologias integradoras já citadas.

Os objetivos decorrentes dos princípios norteadores supracitados apontam, então, ora para melhorar o desempenho do sistema, no caso dos sistemas funcionalistas e estruturalistas, ora para incluir as diferentes visões de realidade dos diversos atores do sistema, no caso dos sistemas interpretativistas e críticos, e tendo, no caso destes últimos, a emancipação e liberdade dos indivíduos em situação de opressão. A existência de bases filosóficas, conceituais e metodológicas integrativas mostra que estes objetivos podem ser considerados de forma inclusiva ao se abordar um sistema que seja complexo o suficiente para abarcar ambas as características.

Os objetivos quanto aos sistemas referem-se também aos tipos de problemas neles encontrados, os quais variam, dependendo do nível de ordem interna e dos ciclos de causa-efeito envolvidos, entre aqueles que podem ser resolvidos por meio de intervenções eminentemente técnicas (no caso dos sistemas simples e complicados) até aquelas marcadas pela complexidade decorrente das interações sociais, próprias dos sistemas interpretativistas e críticos. Também estes tipos de problemas podem ser abordados a partir de uma perspectiva integrativa no caso de sistemas complexos.

As metodologias disponíveis para se abordar de forma adequada cada um dos tipos de sistema são as mais variadas, incluindo desde aquelas bastante tradicionais em sistemas técnicos como a Hard Systems Engineering, ou as que facilitam a compreensão da estrutura dos sistemas como a System Dynamics, até aquelas voltadas à compreensão de sistemas sociais e/ou sociotécnicos, como a SSM de Checkland e a CST e CSH. Cada uma destas metodologias oferece um conjunto próprio de métodos e ferramentas que permitem operacionalizá-las, as quais podem inclusive ser utilizadas a partir de uma perspectiva integrada, variada e não-excludente presente em metodologias como a TSI e a Multimetodologia.

A partir desta ampla variedade de se ver e abordar sistemas pode-se identificar também diversas contribuições possíveis para estudos e projetos em Educação Ambiental, uma vez que não há uma mais correta ou verdadeira do que a outra. Para que cada uma delas seja efetiva em EA, deve-se antes analisar o objeto de estudo a partir de cada uma das categorias colocadas anteriormente, seja em um nível mais conceitual e teórico, questionando os paradigmas filosóficos, os aspectos ontológicos e epistemológicos, os princípios norteadores e o objetivo do estudo, para então considerar-se quais as metodologias, métodos e ferramentas mais adequados para cada situação. 
As contribuições podem então variar desde a compreensão de aspectos mais objetivos do sistema (como aqueles de natureza técnica, econômica ou ambiental) a fim de melhorar seu desempenho, oferecida pelas perspectivas funcionalistas e estruturalistas, até o entendimento dos aspectos humanos, tanto individuais quanto coletivos, em termos da sua relação com a tecnologia, a economia e o meio natural, a fim de se promover mudanças de forma participativa e colaborativa. Estas mudanças podem incluir, inclusive, uma maior emancipação dos próprios indivíduos envolvidos naquele sistema. Pode-se ainda, no caso de sistemas que incluam aspectos objetivos quanto subjetivos, adotar-se uma práxis integrativa que possibilite a compreensão integrada destes aspectos.

Neste sentido, espera-se que a síntese aqui apresentada possa fornecer àqueles que estudam o tema da Educação Ambiental, fornecendo-Ihes uma estrutura teórico-metodológica que possibilite tanto um melhor enquadramento dos estudos quanto uma melhor escolha das estratégias mais adequadas de abordagem.

\section{Agradecimentos}

Ao Conselho Nacional de Desenvolvimento Científico e Tecnológico (CNPq), pelo apoio financeiro à pesquisa; à Universidade Estadual de Londrina (UEL), que possibilitou o pós-doutorado do autor na temática abordada neste artigo.

\section{Referências}

ALMAN, D. Multilevel System Analysis: An introduction to Systems, n. November 2013.

BRASIL. Lei no 9.795, de 27 de abril de 1999. Institui a Política Nacional de Educação Ambiental, e dá outras providências. Diário Oficial [da] República Federativa do Brasil, Brasília, DF, 28 abr. 1999. Disponível em: $<$ http://www.planalto.gov.br/ccivil 03/leis/19795.htm>. Acesso em: agosto de 2019.

CHECKLAND, P.; SCHOLES J. Soft systems methodology in action. Wiley: Chichester, 1990.

MILLER, J. H.; PAGE, S. E. Complex Adaptive Systems. Princeton University Press, (March), 431-436. 2007. Disponível em <https://doi.org/10.1016/S14601567(08)10011-3> Acesso em 11 mar 2019.

FLOOD, R.; JACKSON, M. Critical Systems Thinking-Directed Readings. $347 \mathrm{pp}$. New York: Wiley. [Collection of the most important articles about history, methodology, and application of the critical systems approach.] 1991.

FORRESTER, JAY, W. Counterintuitive Behavior of Social Systems. Theory and Decision, v. 2, n. 2, p. 109-140, 1971. 
ISON, R. Systems Practice: How to Act in a Climate-Change World. London: Springer, 2010.

KIM, D. H. System Archetypes I. Pegasus Communications, Inc., 1992.

LAZLO, A., KRIPPNER, S. Systems Theories: Their Origins, Foundations, and Development. In: J.S. Jordan (Ed.), Systems Theories and A Priori Aspects of Perception. Amsterdam: Elsevier Science. Ch. 3, pp. 47-74. 1998.

MEADOWS, D. Places to Intervene in a System by Donella Meadows. World, v. 91, n. winter, p. 21, 1999. Disponível em: <http://www.biomimicryinstitute.org/ downloads/PlacesTolntervenelnTheSystem.pdf>. Acesso em 14 mar 2019.

MEADOWS, D. H.; MEADOWS, D. L.; RANDERS, J.; BEHRENS III, W. W. The Limits to Growth; A Report for the Club of Rome's Project on the Predicament of Mankind. New York: Universe Books. ISBN 0876631650. 1972.

MINGERS, J.; BROCKLESBY, J. Multimethodology: Towards a framework for mixing methodologies. Omega, 25(5), 489-509. 1997.

MINGERS, J.; WHITE, L. (2010). A review of the recent contribution of systems thinking to operational research and management science. European Journal of Operational Research, 207(3), 1147-1161. 2010.

MURPHY, R. Sustainability: A Wicked Problem. Sociologica (ISSN 1971-8853) Fascicolo 2, maggio-agosto 2012.

PORTER, T.; CÓRDOBA, J. Three views of systems theories and their implications for sustainability education. Journal of Management Education, v. 33, n. 3, p. 323-347, 2009.

RITTEL, H.W.J.; WEBBER, M.M. Dilemmas in a general theory of planning. Policy Sci, v.4: n.155, 1973.

SAMPAIO, C.P. Pensamento de sistemas e design thinking aplicados a wicked problems: sistema de coleta seletiva de resíduos sólidos urbanos. Anais do I Seminário Interdisciplinar de Pesquisas em Resíduos - SIPR, pp. 59-63. Londrina: Rede de Pesquisas em Resíduos da UEL/RPR.UEL, 2019.

SIMON, H. A. The Sciences of the Artificial, Cambridge: MIT Press. 1969.

SNOWDEN, D. J. Multi-ontology sense making: A new simplicity in decision making. Informatics in Primary Care, v. 13, n.1, p.45-53. 2005.

ULRICH, W. Critical Heuristics of Social Planning: A New Approach to Practical Philosophy. Bern, Switzerland, and Stuttgart, Germany: Haupt. Paperback reprint version, Chichester, UK: Wiley, 1994,same pagination, 1983.

ULRICH, W.; REYNOLDS, M. Critical systems heuristics. In: Reynolds, Martin and Holwell, Sue eds. Systems Approaches to Managing Change: A Practical Guide. London: Springer, pp. 243-292. 2010.

VANDENBROECK, B. Systems Thinking and Four Forms of Complexity. Antwerpen: Shiftn, 2015. 